\title{
Effects of neutron radiation on Nrf2-regulated antioxidant defense systems in rat lens
}

\author{
YUEQIN CHEN ${ }^{1,2^{*}}$, JUNDONG FENG $^{3 *}$, JINGYU LIU $^{2}$, HAO ZHOU $^{3}$, \\ HUIYAO LUO ${ }^{3}$, CHUNYAN XUE $^{2}$ and WEIPING GAO ${ }^{1}$
}

\begin{abstract}
${ }^{1}$ Department of Ophthalmology, The Affiliated Hospital of Nanjing University of Chinese Medicine, Nanjing, Jiangsu 210029; ${ }^{2}$ Department of Ophthalmology, Jinling Hospital, School of Medicine, Nanjing University, Nanjing, Jiangsu 210002; ${ }^{3}$ Key Laboratory of Nuclear Technology Application and Radiation Protection in Astronautics (Nanjing University of Aeronautics and Astronautics), Ministry of Industry and Information Technology, Department of Nuclear Science and Technology, Nanjing University of Aeronautics and Astronautics, Nanjing, Jiangsu 210016, P.R. China
\end{abstract}

Received Augsut 25, 2020; Accepted January 8, 2021

DOI: $10.3892 /$ etm.2021.9765

\begin{abstract}
Accumulating evidence suggests that ionizing radiation (IR)-induced cataract may be associated with oxidative stress. Nuclear factor erythroid 2-related factor 2 (Nrf2) serves as a master regulator of the antioxidant defense system against oxidative stress. The present study aimed to investigate the effects of different doses of neutron radiation on the Nrf2-reegulated antioxidant defense system in rat lens and assess the status of oxidative stress. A total of $24 \mathrm{SD}$ rats were randomly divided into the following four groups: i) Control group; iis) $0.4 \mathrm{~Sv}$ group; iii) $1.2 \mathrm{~Sv}$ group; and iv) $3.6 \mathrm{~Sv}$ group. The rats were sacrificed 7 days after radiation and lenses were dissected for histological, biochemical (malondialdehyde, glutathione and superoxide dismutase) and western blot (Nrf2, glutamate-cysteine ligase catalytic subunit and heme oxygenase 1) analyses. The morphological features of the lenses remained intact in the $0.4 \mathrm{~Sv}, 1.2 \mathrm{~Sv}$ and control groups, whilst the lenses in the $3.6 \mathrm{~Sv}$ group exhibited injuries. Results from the TUNEL assay demonstrated apparent apoptosis in lens epithelial cells following 3.6 Sv neutron radiation whereas sparse apoptosis was observed following $0.4 \mathrm{~Sv}$ and
\end{abstract}

Correspondence to: Dr Chunyan Xue, Department of Ophthalmology, Jinling Hospital, School of Medicine, Nanjing University, 305 East Zhongshan Road, Nanjing, Jiangsu 210002, P.R. China

E-mail: xuechunyancn@126.com

Dr Weiping Gao, Department of Ophthalmology, The Affiliated Hospital of Nanjing University of Chinese Medicine, 155 Hanzhong Road, Nanjing, Jiangsu 210029, P.R. China

E-mail: gao-weiping@163.com

*Contributed equally

Key words: ionizing radiation, lens, oxidative stress, nuclear factor erythroid 2-related factor 2, glutamate-cysteine ligase catalytic subunit, heme oxygenase-1
1.2 Sv radiation. Malondialdehyde levels were reduced in the $0.4 \mathrm{~Sv}$ and $1.2 \mathrm{~Sv}$ groups but increased in the $3.6 \mathrm{~Sv}$ group, compared with those in the control group. Conversely, glutathione expression and the activity of superoxide dismutase were higher in the $0.4 \mathrm{~Sv}$ and $1.2 \mathrm{~Sv}$ groups, but lower in the 3.6 Sv group, compared with those in the control group. In addition, the total and nuclear protein levels of Nrf2 were increased following neutron radiation compared with those in the control group, though the Nrf2 protein levels decreased in the $3.6 \mathrm{~Sv}$ group compared with those in the $1.2 \mathrm{~Sv}$ group. The levels of glutamate-cysteine ligase catalytic subunit and heme oxygenase 1, downstream antioxidant enzymes of Nrf2, demonstrated the same profile as that in Nrf2. Taken together, the results of the present study suggest that neutron radiation affects Nrf2-regulated antioxidant systems in a two-stage process. Namely, the induction phase for low-dose radiation and regression phase for high-dose radiation. Therefore, it was hypothesized that activation and enhancement of the Nrf2-regulated antioxidant system may be useful in preventing or delaying IR-induced cataract, which may be extended even for other diseases associated with oxidative stress.

\section{Introduction}

It is well-known that reactive oxygen species (ROS) serve a critical role in a number of biological processes, including disease and aging (1). Oxidative stress is defined as cellular damage from ROS exposure that occurs when ROS production exceeds the capability of the cellular antioxidant defense system $(1,2)$. This ultimately leads to the modification and degradation of proteins, damage to the mitochondria and cell death $(1,2)$. Ionizing radiation (IR) causes damage to biological tissues by inducing ROS production and can cause oxidative injury to cellular macromolecules like DNA, lipids and proteins, resulting in the impairment of organs and systems and even mortality $(3,4)$. IR can arise from occupational exposure, medical procedures or exposure to nuclear explosion (5-7).

The lens is an avascular, encapsulated and transparent tissue containing organelle-free, terminally differentiated fiber 
cells at the center, where a single layer of epithelial cells covers the anterior surface of the organ (8). The lens is one of the most radiosensitive tissues in the body, where the dividing epithelial cells near the equator serve a critical role in IR-induced cataract (9). ROS-induced by IR can cause oxidative damage to proteins, resulting in protein aggregation and cataract, which is the leading cause of blindness worldwide (1). However, the lens has evolved several antioxidant systems to defend against ROS damage, including ROS scavenger systems and enzyme protective systems (1). Several antioxidant enzymes, such as superoxide dismutase (SOD) and catalase, are regulated by the key transcription factor, nuclear factor erythroid 2-related factor 2 (Nrf2) (10). Nrf2 is normally sequestered by the Kelch-like ECH-associated protein 1 (Keap1) protein in the cytoplasm, which serves as a master regulator of the antioxidant response element (ARE)-driven cellular defense system against oxidative stress (10). Upon activation by ROS, the Nrf2-Keap1 complex is disrupted and the free Nrf2 subsequently translocates into the nucleus and binds to ARE, which in turn activates the expression of downstream antioxidant and detoxification genes to combat ROS and boost cell survival (11). Previous studies have reported that the onset of cataract as a result of IR may be associated with compromised antioxidant capacity in the lens $(12,13)$. However, the effects of varying IR doses on the Nrf2-regulated antioxidant defense systems of lens and underlying molecular mechanisms remain poorly understood.

In present study, animal models were used to simulate ocular injury caused by space radiation experienced by astronauts whilst participating in missions on the International Space Station (ISS). For an ISS-type orbit, estimates of neutron contribution to an astronaut's total radiation dose range is $30-60 \%$ (14). The quality factor for the neutron tends to be $4-5 \mathrm{X}$ greater compared with that in the charged particles from space radiation (15). Therefore, the impact of neutron radiation on ocular injuries in space travel serves an important role. Furthermore, little has been investigated regarding neutron radiation on the lens. Therefore, the present study chose neutrons as the radiation source, which aimed to investigate the effects of different doses of neutron radiation on the status of the Nrf2 antioxidant defense system and severity of oxidative stress in rat lenses in vivo. Results from the present study hopes to provide a deeper understanding of the effects of neutron radiation on the lens and the role of Nrf2 in the regulation of the antioxidant defense systems following radiation, which is indispensable for the prevention and treatment of IR-induced cataract. In addition, it is hoped that data from the present study facilitate the development of management strategies for even other oxidative stress-associated diseases and the field of radiation protection.

\section{Materials and methods}

Animals and neutron radiation. A total of 24 male

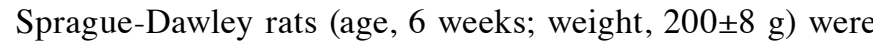
purchased from the Central Animal House of Qinglongshan Institute (Nanjing, China). All procedures involving animals and corresponding experimental protocols were approved by The Ethics Committee of Jinling Hospital (approval no. 2020 JLHGKJDWLS-109; Nanjing, China). Animals were allowed free access to water and normal pellet diet and were housed in polypropylene cages bedded with sterilized rice husk under 12-h light/dark cycles with a temperature of $24 \pm 1^{\circ} \mathrm{C}$ and humidity of $50 \pm 10 \%$. The rats were randomly divided into the following four groups ( $n=6$ rats in each group): i) Control group; ii) $0.4 \mathrm{~Sv}$ group; iii) $1.2 \mathrm{~Sv}$ group; and iv) $3.6 \mathrm{~Sv}$ group.

Neutron radiations were performed at the Radiological Research Center of Nanjing University of Aeronautics and Astronautics (Nanjing, China). Neutrons were generated by the reaction of deuterium on a tritium target (16), and rats were anesthetized (intraperitoneal injection of $90 \mathrm{mg} / \mathrm{kg}$ ketamine and xylazine $10 \mathrm{mg} / \mathrm{kg}$ ) and immobilized by jigs surrounding the neutron generation device (Fig. 1A-C) (17). Rats in the control group were sham irradiated, that is, rats were immobilized like the other 3 groups but were irradiated by $0 \mathrm{~Sv}$. The eyes of the rats the other three groups were 17, 10 and $6 \mathrm{~cm}$ from the target center (Fig. 1D), where the dose rates were 14,45 and $131 \mathrm{mSv} / \mathrm{h}$, respectively. Radiation was delivered in four fractions in 4 successive days (once per day) and the total doses were $0.4,1.2$ and 3.6 Sv, respectively. Sham-radiated control animals were treated similarly to radiated animals but the radiation source was not activated. The rats were anesthetized with an intraperitoneal injection of pentobarbital sodium (30 mg/kg body weight) and subsequently sacrificed by cervical dislocation 7 days after the final radiation. Their eyes were then enucleated, where their lenses were collected for histopathological, biochemical and western blot analyses.

Histological analysis. The eyeballs were fixed in $10 \%$ formalin for $24 \mathrm{~h}$ at room temperature and dehydrated using a gradient alcohol series (70, 80, 90, 95 and $100 \%$ ethanol) followed by two xylene treatments. The samples were embedded in paraffin and then cut into 5- $\mu$ m-thick sections. Tissue sections were subsequently deparaffinized in xylene and then rehydrated with a gradient alcohol series (100, 95, 80 and 75\% ethanol) and washed in PBS. The sections were then stained with hematoxylin for $7 \mathrm{~min}$ and eosin for $3 \mathrm{~min}$ at room temperature. For histopathological analysis, the slides were observed under a light microscope (magnification, x400; Olympus BX41).

Biochemical assays. The lens tissues were homogenized in cold PBS (10\% w/v) and centrifuged at 3,000 x g for $10 \mathrm{~min}$ at $4^{\circ} \mathrm{C}$. The supernatant was used for biochemical analyses, as previously described (18-20).

Malondialdehyde (MDA) concentration in the homogenate was determined based on the thiobarbituric acid reactive substances assay (TBARS), using the MDA Assay kit (cat. no. A003-1; Nanjing Jiancheng Bioengineering Institute). Briefly, TBA reacted with MDA to form red products, the absorbance of which can be measured at a wavelength of $532 \mathrm{~nm}$. MDA concentration was expressed as nmol of MDA per milligram of protein ( $\mathrm{nmol} / \mathrm{mg}$ protein).

Reduced glutathione (GSH) concentration was analyzed based on the dithionitrobenzoic acid (DTNB) reaction, using the GSH Assay kit (Nanjing Jiancheng Bioengineering Institute; cat. no. A006-2-1). DTNB reacts with reduced GSH to form yellow products, where the absorbance of which was measured at a wavelength of $405 \mathrm{~nm}$. GSH concentration was expressed as $\mu \mathrm{g}$ of GSH per milligram of protein $(\mu \mathrm{g} / \mathrm{mg}$ protein). 
A

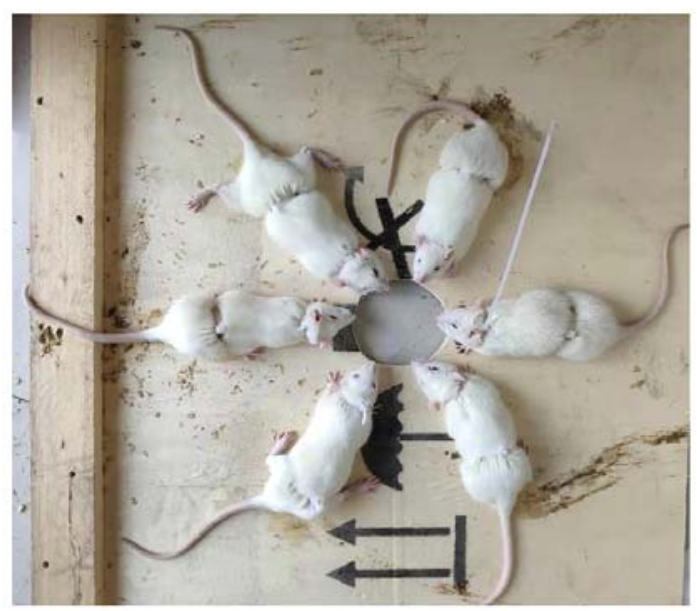

C

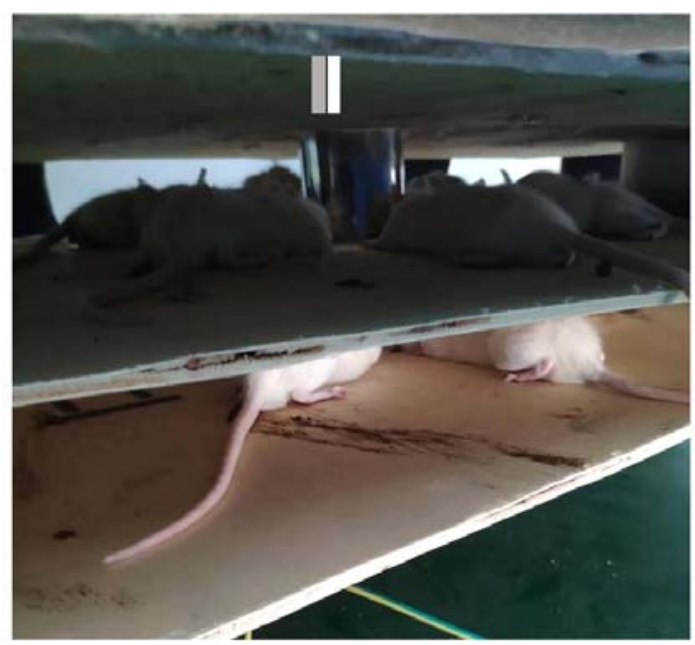

B

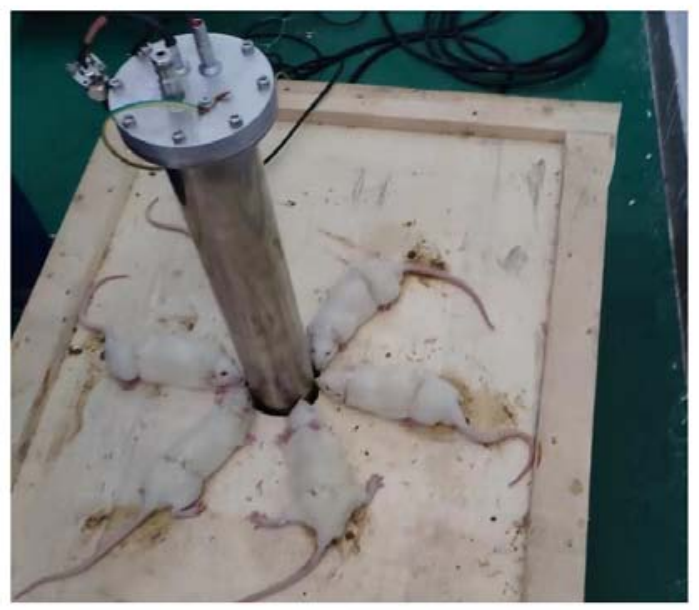

D

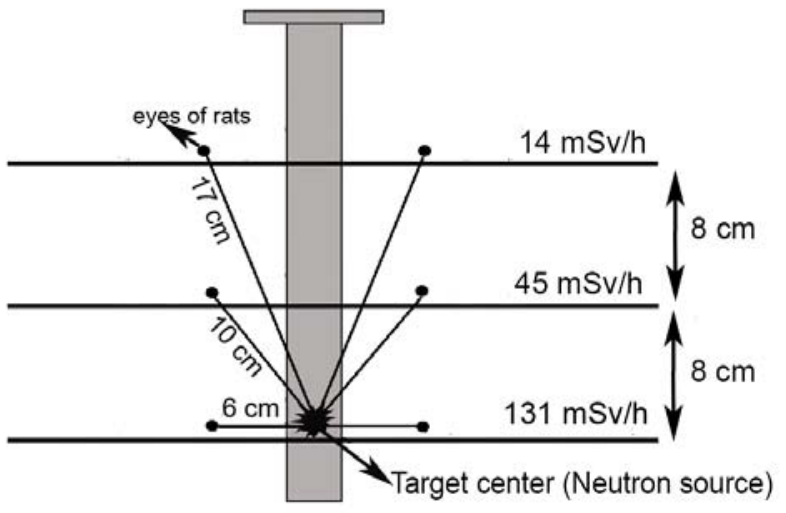

Figure 1. Diagram of the neutron radiation of rats. Rats were exposed to neutron radiation. (A) Bird's eye, (B) vertical and (C) lateral view of the irradiation apparatus in the presence of the neutron generator. (D) The eyes of the rats were 17,10 and $6 \mathrm{~cm}$ from the radiation source in the $0.4,1.2$ and $3.6 \mathrm{~Sv}$ groups, and the dose rates were 14,45 and $131 \mathrm{mSv} / \mathrm{h}$, respectively (longitudinal section of relative position for rats' eyes and neutron generator tube). Solid black spots represent the position of the rats' eyes.

Superoxide dismutase (SOD) activity was assessed using the SOD Assay kit (cat. no. A001-3; Nanjing Jiancheng Bioengineering Institute), based on the xanthine and xanthine oxidase systems, where absorbance was measured at a wavelength of $450 \mathrm{~nm}$. In total, 1 unit (U) of SOD activity was defined as the amount of enzyme causing 50\% inhibition of the xanthine and xanthine oxidase reaction systems. SOD activity was expressed as units per milligram protein (U/mg protein).

Western blotting. Total protein and nuclear protein of lens tissue were extracted using the Whole Cell Lysis Assay (cat. no. KGP250/KGP2100) and Nuclear Protein Extraction (cat. no. KGP150/KGP1100) kits (both from Nanjing KeyGen Biotech Co., Ltd.), according to the manufacturer's protocols. For total protein extraction, lenses were homogenized in ice-cold lysis buffer to obtain tissue homogenate. The homogenate was subsequently centrifuged at $12,000 \mathrm{x}$ g for $5 \mathrm{~min}$ at $4^{\circ} \mathrm{C}$ and the supernatant was collected. For nuclear protein extraction, lenses were homogenized in ice-cold lysis buffer and the homogenate was centrifuged at 3,000 $\mathrm{xg}$ for $10 \mathrm{~min}$ at $4^{\circ} \mathrm{C}$. Following centrifugation, the pellets were sonicated $\left(3,000 \mathrm{rpm}\right.$ for $15 \mathrm{sec}$ at $\left.4^{\circ} \mathrm{C}\right)$ with appropriate volumes of nuclear extraction buffer and re-centrifuged at $14,000 \mathrm{x} \mathrm{g}$ for $30 \mathrm{~min}$ at $4^{\circ} \mathrm{C}$. The supernatant was collected for nuclear fractions. The protein samples were quantified using bicinchoninic acid kit (cat. no. KGPBCA; Nanjing KeyGen Biotech Co., Ltd.). In total, $50 \mu \mathrm{g}$ protein samples were separated by $10 \%$ SDS-PAGE and transferred onto polyvinylidene fluoride membranes (EMD Millipore). The membranes were blocked with 5\% non-fat milk in TBST for $2 \mathrm{~h}$ at room temperature and incubated with primary antibodies at $4^{\circ} \mathrm{Covernightagainst:} \mathrm{Nrf} 2$ (1:1,000; Abcam; cat. no. ab137550), glutamate-cysteine ligase catalytic subunit (GCLC; 1:1,000; Abcam; cat. no. ab207777), heme oxygenase 1 (HO-1; 1:1,000; Abcam; cat. no. ab189491), $\beta$-actin (1:5,000; Abcam; cat. no. ab8226) and Histone H2A (1:1,000; Abcam; cat. no. ab177312). Following primary antibody incubation, membranes were incubated with goat anti-rabbit HRP-conjugated secondary antibody (1:10,000; cat. no. BL003A; Biosharp) for $1 \mathrm{~h}$ at $37^{\circ} \mathrm{C}$. Protein bands were visualized with New Super ECL (cat. no. KGP1127-KGP1128; 

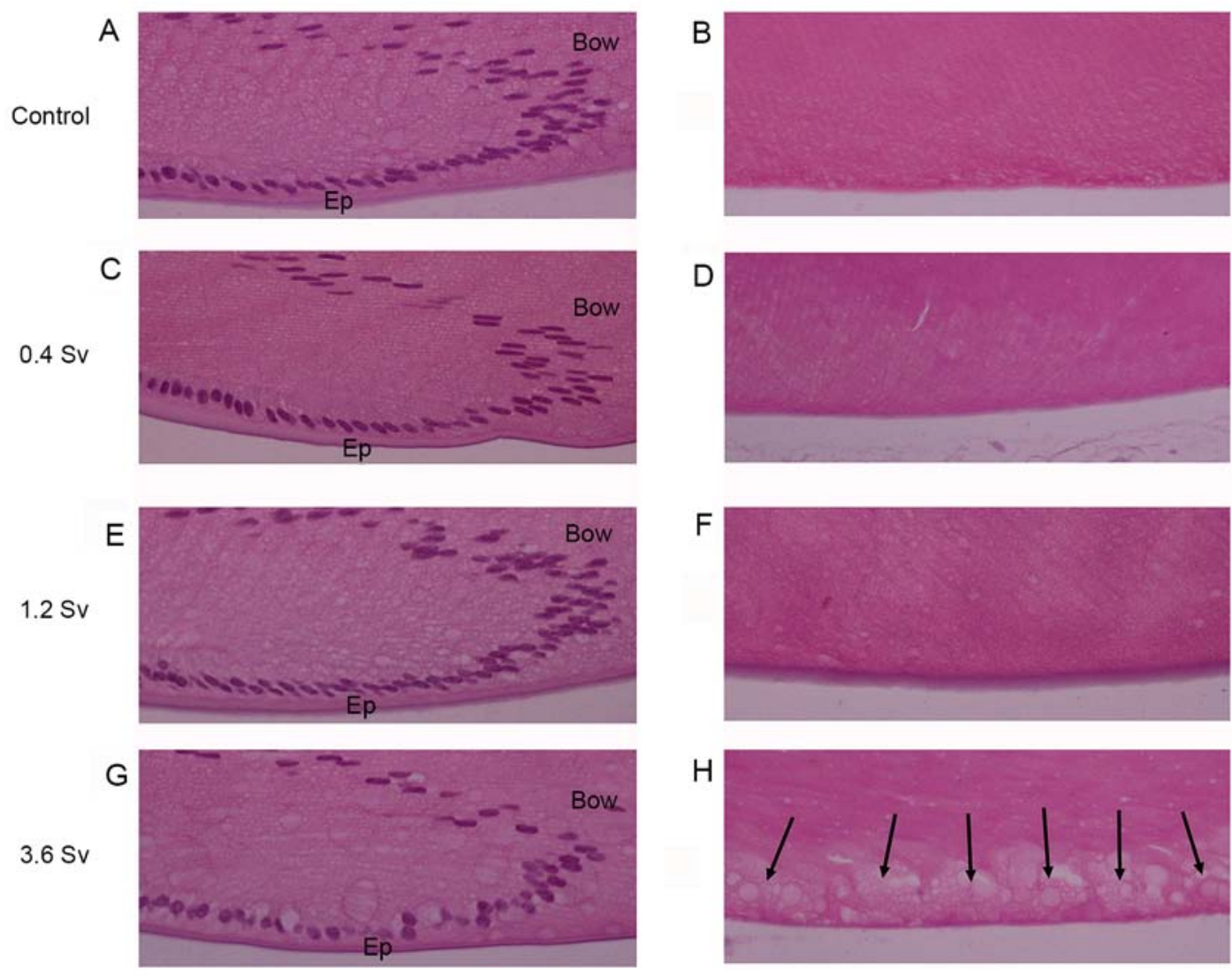

Figure 2. Histological images of the lenses following neutron radiation. The lens tissue sections were stained with hematoxylin and eosin and observed under a light microscope (magnification, x400). Lens bow region in the (A) control, (C) 0.4 Sv, (E) 1.2 Sv and (G) 3.6 Sv groups. Lens posterior pole region in (B) control, (D) 0.4 Sv, (F) 1.2 Sv and (H) 3.6 Sv groups. Black arrows indicate vacuolization under the posterior capsule. Ep, epithelial cells. Bow, lens bow region.

Nanjing KeyGen Biotech Co., Ltd.) using G:BOX chemiXR5 (Syngene Europe), whilst the densities were determined using ImageJ software (version 2.1.4.7; National Institutes of Health). The band densities of each sample were normalized to $\beta$-actin or Histone H2A.

Terminal deoxynucleotidyl transferase dUTP nick-end labeling (TUNEL) assay. The eyeballs were fixed in $10 \%$ formalin for $24 \mathrm{~h}$ at room temperature, paraffin-embedded, then sectioned at a thickness of $5 \mu \mathrm{m}$. TUNEL analysis was performed using One Step TUNEL Apoptosis Assay Kit (cat. no. KGA7071; Nanjing KeyGen Biotech Co., Ltd.), according to the manufacturer's protocol. Briefly, paraffin-embedded lens sections were deparaffinized in xylene, rehydrated with a gradient ethanol series, digested with protein $\mathrm{K}$ for $30 \mathrm{~min}$ at $37^{\circ} \mathrm{C}$ and incubated with the TUNEL reaction mixture for $1 \mathrm{~h}$ at $37^{\circ} \mathrm{C}$. Nuclei were counterstained with $2 \mu \mathrm{g} / \mathrm{ml}$ DAPI (cat. no. KGA215; Nanjing KeyGen Biotech Co., Ltd.) in the dark for $5 \mathrm{~min}$ at room temperature, and then rinsed with PBS. Antifade Mounting Medium (cat. no. KGF028; Nanjing KeyGen Biotech Co., Ltd.) was used for mounting. TUNEL-positive cells were identified via green fluorescence. For quantitative analysis, the number of lens epithelial cell nuclei and the number of TUNEL-positive epithelial nuclei were manually counted. The TUNEL index was expressed as the percentage of the number of TUNEL-positive epithelial cells in relation to the total number of epithelial cells in five randomly selected fields under a fluorescence microscope (magnification, x200), as previously described (21).

Statistical analysis. Statistical analysis was performed using SPSS 17.0 software (SPSS, Inc.). All experiments were performed in triplicate and data are presented as the mean \pm standard deviation. One-way ANOVA was used to compare differences among multiple groups followed by Tukey's post hoc test, whilst the unpaired Student's t-test was used to compare differences between two groups. $\mathrm{P}<0.05$ was considered to indicate a statistically significant difference.

\section{Results}

Effects of neutron radiation on lens morphology. The morphological features of the lenses remained intact in the $0.4 \mathrm{~Sv}$, 1.2 Sv and control groups, including the epithelial cells, lens bow pattern, fiber cells and posterior pole (Fig. 2). However, lenses that were exposed to $3.6 \mathrm{~Sv}$ exhibited injury, with reduced density and abnormal alignments in the epithelial cells, slight distortion in the lens bow configuration, swollen cortical fibers and vacuolization near the posterior pole of the lens (Fig. 2).

Effects of neutron radiation on oxidative stress and SOD activity in rat lens. Compared with those in the control group, 
A

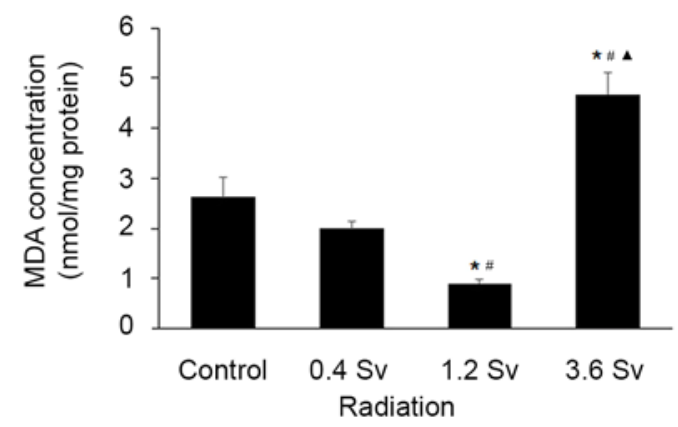

C

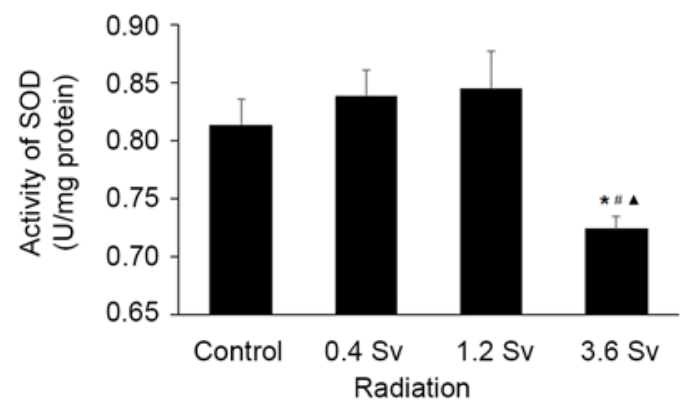

B

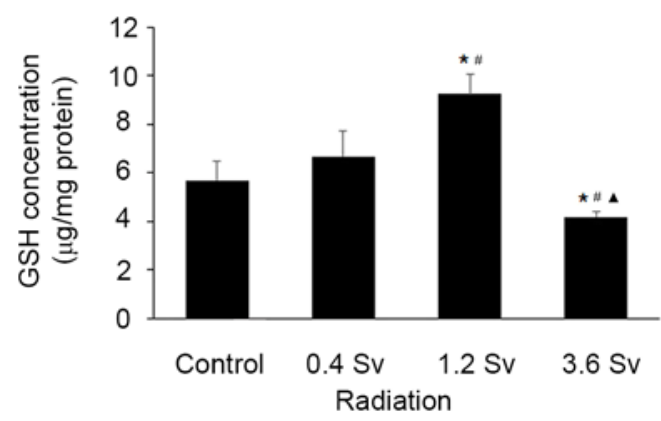

Figure 3. Oxidative stress and SOD activity in rat lenses following neutron radiation. (A) MDA concentration was determined based on thiobarbituric acid reaction. (B) GSH concentration was analyzed based on the dithionitrobenzoic acid reaction. (C) SOD activity was assessed using the xanthine and xanthine oxidase systems. ${ }^{*} \mathrm{P}<0.05$ vs. Control; ${ }^{\#} \mathrm{P}<0.05$ vs. $0.4 \mathrm{~Sv} ;{ }^{\wedge} \mathrm{P}<0.05$ vs. $1.2 \mathrm{~Sv}$. SOD, superoxide dismutase; MDA, malondialdehyde; GSH, glutathione.

MDA levels were found to be significantly lower in the $1.2 \mathrm{~Sv}$ group $(\mathrm{P}<0.05$; Fig. $3 \mathrm{~A})$ but significantly higher in the $3.6 \mathrm{~Sv}$ group $(\mathrm{P}<0.05$; Fig. 3A).

Conversely, GSH levels were significantly increased in the 1.2 Sv groups $(\mathrm{P}<0.05$; Fig. $3 \mathrm{~B})$ and significantly decreased in the $3.6 \mathrm{~Sv}$ group, compared with those in the control group $(\mathrm{P}<0.05$; Fig. 3B).

Although SOD activity was increased in the $0.4 \mathrm{~Sv}$ and 1.2 Sv groups, the differences was not found to be statistically significant (Fig. 3C). Conversely, SOD activity was significantly decreased in the $3.6 \mathrm{~Sv}$ group compared with that in the control group $(\mathrm{P}<0.05$; Fig. 3C).

Effects of neutron radiation on $\mathrm{Nrf} 2$ and downstream antioxidant enzymes. The total and nuclear protein levels of Nrf2 were increased following $0.4,1.2$ and $3.6 \mathrm{~Sv}$ neutron radiation compared with the control group (all $\mathrm{P}<0.05$; Fig. 4). However, the levels were significantly lower in the $3.6 \mathrm{~Sv}$ group compared with that in the $1.2 \mathrm{~Sv}$ group $(\mathrm{P}<0.05$; Fig. $4 \mathrm{C}$ and $\mathrm{D})$. The protein levels of HO-1 and GCLC, downstream antioxidant enzymes of Nrf2 (4), exhibited similar trends $(\mathrm{P}<0.05$ in the $1.2 \mathrm{~Sv}$ group vs. control group for $\mathrm{HO}-1 ; \mathrm{P}<0.05$ in the $0.4 \mathrm{~Sv}$ and 1.2 Sv groups vs. control group for GCLC; Fig. 4E and F).

Apoptosis of lens epithelial cells. The results of the TUNEL assay demonstrated that the apoptotic cells were sparse following radiation with $0.4 \mathrm{~Sv}$ and $1.2 \mathrm{~Sv}$ (Fig. 5B and C). However, 3.6 Sv neutron radiation significantly induced cell apoptosis compared with that in control (Fig. 5D and E). In addition, quantitative assessment demonstrated that the TUNEL index was significantly higher following radiation with 3.6 Sv compared with that in the control group $(\mathrm{P}<0.05$; Fig. 5E). Notably, the difference was not statistically significant following radiation with $0.4 \mathrm{~Sv}$ and $1.2 \mathrm{~Sv}$ compared with that in the control group (Fig. 5E).

\section{Discussion}

IR-induced damage is primarily attributed to ROS, which serves an important role in the effects of radiation on biological tissues and organisms (22). Organisms have antioxidant defense systems for scavenging ROS, which are reported to be regulated by the Nrf2/ARE signaling pathway $(10,23)$. The present study investigated the effect of neutron radiation on the status of oxidative stress and Nrf2-regulated antioxidant defense systems in rat lenses at different radiation doses $(0.4$, 1.2 and $3.6 \mathrm{~Sv})$.

Several protective systems have evolved in the ocular lens, including the antioxidant enzymatic and nonenzymatic systems, to combat oxidative stress induced by ROS (1). SOD is one of these antioxidant enzymes. When the SOD activity increases, the capability of eliminating ROS enhances (24). MDA is commonly used to assess the active oxygen damage (25). In the present study, SOD activity increased following radiation with 0.4 and $1.2 \mathrm{~Sv}$ compared with that in the control group, but the difference was not statistically significant. Conversely, MDA levels were reduced in the 0.4 and 1.2 Sv groups compared with those in the control group. This phenomenon of beneficial biological effects caused by low-dose radiation was previously termed as 'radiation hormesis' (26). A number of previous studies have addressed the beneficial effects of low-dose radiation, which demonstrated 
A

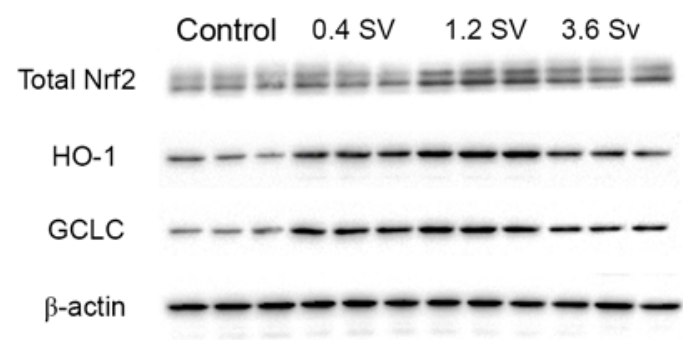

C

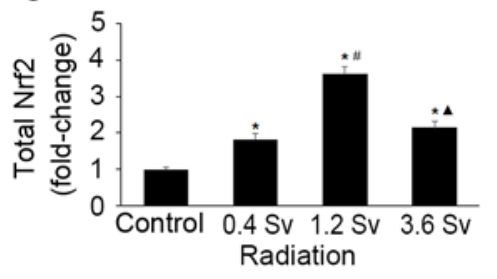

$\mathrm{E}$

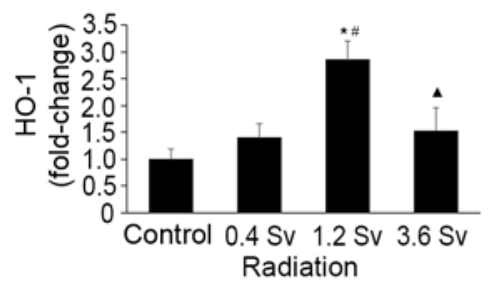

B

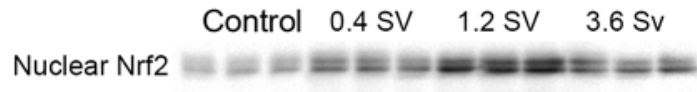

Histone $\mathrm{H} 2 \mathrm{~A}-0-0-0-0-1-0$

$\mathrm{D}$
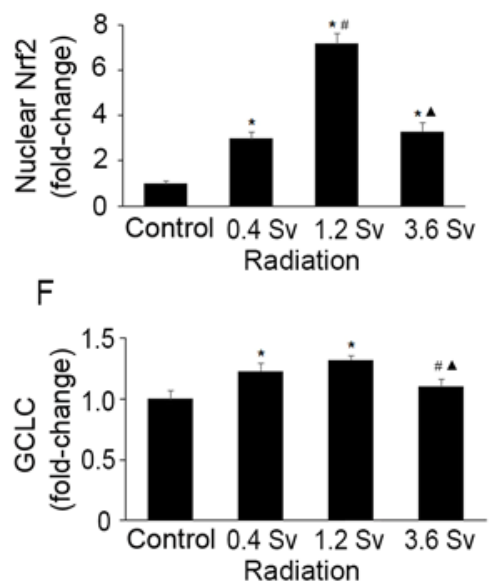

Figure 4. Protein levels of Nrf2, HO-1 and GCLC in rat lenses following neutron radiation. (A) Western blot analysis was performed to detect protein levels of total Nrf2, HO-1 and GCLC. Each signal was normalized to that of $\beta$-actin. (B) Western blot analysis was performed to measure nuclear Nrf2 expression, where the signal was normalized to that from Histone H2A. Quantification of (C) total Nrf2, (D) nuclear Nrf2, (E) HO-1 and (F) GCLC. For (C-F), all values were normalized to that of control for each protein. ${ }^{*} \mathrm{P}<0.05$ vs. Control; ${ }^{*} \mathrm{P}<0.05$ vs. $0.4 \mathrm{~Sv} ;{ }^{\wedge} \mathrm{P}<0.05$ vs. $1.2 \mathrm{~Sv}$. Nrf2, nuclear factor erythroid 2 -related factor 2 ; HO-1, heme oxygenase 1; GCLC, glutamate-cysteine ligase catalytic subunit.

induction of antioxidant enzymes by low-dose radiation both in vitro and in vivo. For example, Yamaoka et al (24) assessed changes in SOD activity and lipid peroxide (TBARS/MDA) levels in brain, lungs, liver, thymus, spleen and bone marrow of rats following exposure to whole-body low-dose X-radiation, which reported that SOD activity increased in immune organs following exposure to radiation at doses of 0.05-0.50 Gy for $4 \mathrm{~h}$, whilst the levels of MDA reduced. In another study, Pathak et al (27) demonstrated that SOD activity increased in rodent kidneys by $37 \%$ following exposure to whole-body low-dose $\gamma$-radiation (10-50 cGy) for $12 \mathrm{~h}$. However, the levels of MDA were enhanced, which differed from the results of the present study and the study by Yamaoka et al (24). This variation may be due to species differences in each animal model, radiation source, radiation dose, dose rate, time and organs selected for the measurement of several parameters.

The lens also has a non-enzymatic antioxidant defense system to cope with oxidative stress (28). One aspect of this mechanism is by redox balancing by GSH (28). Lenses contain high concentrations of GSH, which maintain the thiol groups in their reduced forms (29). Reduced GSH levels have been reported in human lens following aging and those with cataract (29). GSH serves a vital role in the maintenance of cellular redox balance by acting as a radical scavenger (30). Yamaoka et al (24) previously demonstrated that GSH levels significantly increased in the kidneys following radiation at varying doses (10-50 cGy) for $12 \mathrm{~h}$. Consistent with these findings, the results of the present study demonstrated that GSH levels were higher in the lower dose groups (0.4 Sv and $1.2 \mathrm{~Sv})$ compared with those in the control group. This increase in GSH levels may be due to the activation of protective responses in the lenses to counteract ROS accumulation (28). However, GSH levels and SOD activity were reduced in the lenses of the high dose group, whilst the MDA levels increased. Taken together, these results suggest that low-dose neutron radiation can increase both the non-enzymatic and enzymatic antioxidant defense systems to overcome IR-induced ROS in the lenses; as the radiation dose increases and ROS content exceeds the capacity of the antioxidant defense systems, lenses may become damaged eventually leading to cataractogenesis. In the present study, the lenses remained intact in the $0.4 \mathrm{~Sv}$, 1.2 Sv and control groups. However, lenses that were exposed to $3.6 \mathrm{~Sv}$ exhibited injury, including reduced density and abnormal alignment of epithelial cells, apoptotic epithelial cells, slight distortion in lens bow, swollen cortical fiber cells and vacuolization near the posterior pole of the lens.

Nrf2 is a redox-sensitive transcription factor that regulates the expression of antioxidant enzymes and phase II metabolic enzymes (31). Nrf2 is normally sequestered in the cytoplasm, which can be activated by signals, such as ROS, which subsequently translocates into the nucleus to regulate expression of downstream antioxidant and detoxificationgenes that 


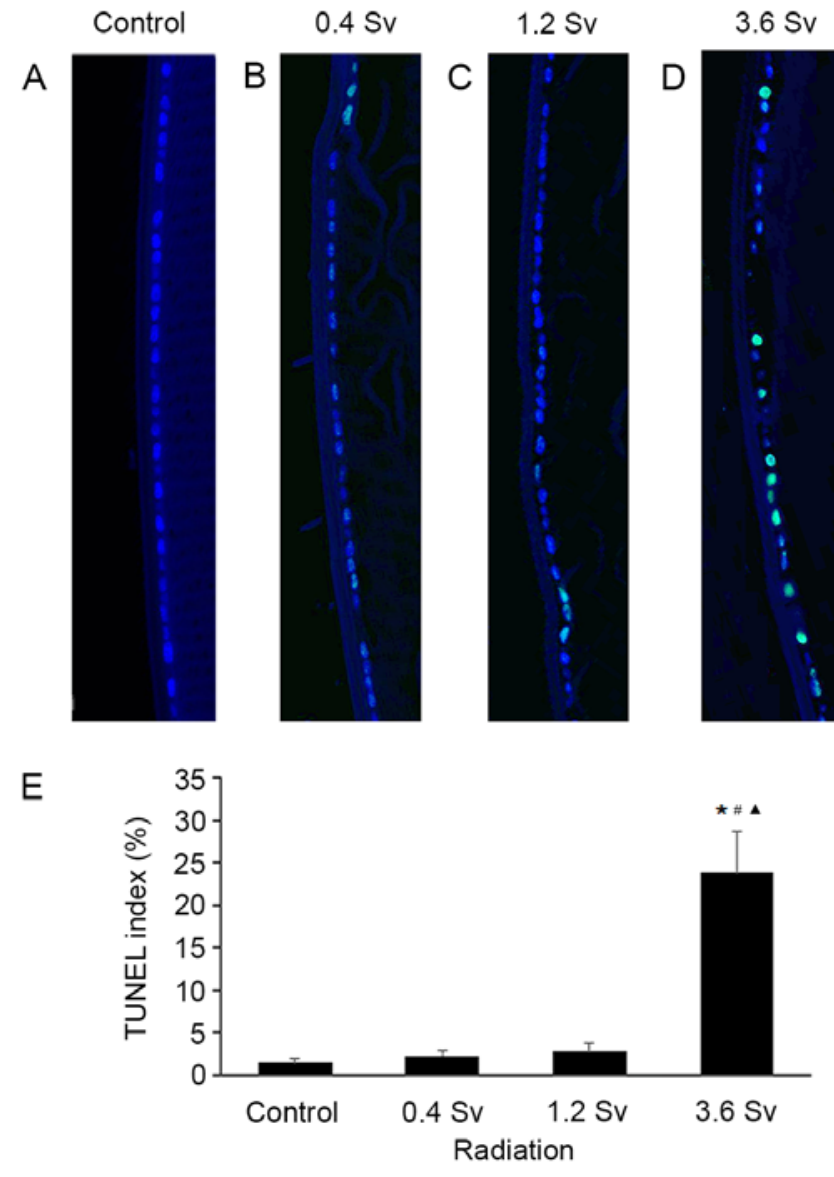

Figure 5. TUNEL staining of rat lens sections following neutron radiation. Representative images of TUNEL-stained sections of lenses from the (A) control, (B) $0.4 \mathrm{~Sv}$, (C) 1.2 Sv and (D) 3.6 Sv groups. Magnification, x200. TUNEL-positive cells were identified using green fluorescence and nucle were counterstained with DAPI (blue). (E) The TUNEL index bar represents the number of TUNEL-positive epithelial cell nuclei/total epithelial cell nuclei in the four groups. ${ }^{*} \mathrm{P}<0.05$ vs. Control; ${ }^{~} \mathrm{P}<0.05$ vs. $0.4 \mathrm{~Sv} ;{ }^{\wedge} \mathrm{P}<0.05$ vs. $1.2 \mathrm{~Sv}$.

counteract ROS (31). These target genes include glutathione peroxidase, SOD, HO-1, quinone oxidoreductase (NQO1) and GCLC $(4,32)$. Activation of the Nrf2 signaling pathway is one of the critical defensive mechanisms against oxidative stress in a number of tissues, including heart, retina, liver and kidney (23). Purbey et al (33) previously reported that Nrf2 activation by ROS is highly selective to radiation exposure compared with other environmental insults, such as a microbial inducer of inflammation. Furthermore, Tsukimoto et al (34) demonstrated that low-dose $\gamma$-radiation induces Nrf2 activation in mouse macrophage RAW264.7 cells, whilst McDonald et al (35) reported that single doses of IR from 2-8 Gy activate ARE-dependent transcription in breast cancer cells. These findings are consistent with the results of the present study, which demonstrated that neutron radiation increased the level of Nrf2 and induced nuclear translocation of Nrf2. Therefore, a two-phase Nrf2 expression was observed following neutron radiation, namely, an increasing phase from 0.4-1.2 Sv and a decreasing phase from 1.2-3.6 Sv. A previous study demonstrated Nrf2-medidated antioxidant defense in two phases in mouse embryonic fibroblasts isolated from p53 and p21 wild-type and knockout pregnant female mice at embryonic day 13 (36). However,
McDonald et al (35) previously demonstrated that radiation activated $\mathrm{Nrf} 2$ in a dose-dependent manner, which differed from the results of the present study. The difference may be due to the different experimental subjects, radiation sources, dose and dose rates.

The results of the present study demonstrated that the levels of the downstream antioxidant enzymes of Nrf2, GCLC and HO-1, increased following radiation at doses of 0.4 and $1.2 \mathrm{~Sv}$. These enzymes serve vital roles in the detoxification and antioxidant processes in the body (37). Zhao et al (38) demonstrated that RNAi-mediated reduction of Nrf2 expression significantly decreases the expression levels of GCLC and HO-1 in radiated lung cancer cells. Notably, Tsukimoto et al (34) reported that HO-1 expression increases following radiation with $>0.1$ Gy of $\gamma$-rays for $24 \mathrm{~h}$ in mouse macrophage RAW264.7 cells. However, the present study demonstrated that high-dose neutron radiation would deplete Nrf2, HO-1 and GCLC protein levels and weaken anti-oxidative stress mechanisms, leading to histopathological changes in the lenses, such as vacuolation under posterior capsule. This is in accordance with the study performed by Liu et al (39), who demonstrated that lower fluences of ultraviolet A rays enhance Nrf2 expression, along with its downstream enzymes HO-1 and NQO1, whilst higher fluences of UVA downregulate Nrf2 and its downstream antioxidant enzymes in corneal endothelial cells.

A limitation of the present study is that neutron radiation [high-linear energy transfer (LET)] was not compared with low-LET radiation, such as X-rays or $\gamma$-rays, or non-ionizing radiation, such as UV. The aim of the present study was to simulate lens injury from space radiation experienced by astronauts participating in missions on the ISS in animal models. Another reason was that, among other types of radiation, neutrons can produce more severe damage compared with $\chi$-rays, $\gamma$-rays or UV (40). A number of studies have studied the effects of other radiations on lens. Bahia et al (41) exposed human lens epithelial cells (HLE) to $\chi$-rays at different doses and demonstrated that HLE exhibits a bi-phasic response in terms of cell viability and ROS. Similar to the Bahia et al study, the findings of the present study also reported a two-phase response. Several studies have demonstrated that excessive UV can induce oxidative damage to the lens and cause cataract $(42,43)$. Similarly, results of the present study demonstrated that high-dose neutron radiation caused lens damage. Another limitation of the present study was that Nrf2-knockout rats were not used. Prospective studies with Nrf2-knockout rats are required to validate the role of the Nrf2 pathway and to identify agents that can prevent or delay IR-induced cataract by activating the Nrf2 pathway.

In conclusion, results of the present study demonstrated that Nrf2-regulated antioxidant systems are affected by neutron radiation in two phases. Low-dose neutron radiation upregulates Nrf2 and its downstream enzymes to combat oxidative damage in the lenses. However, as the radiation dose increases further, Nrf2-mediated antioxidant mechanisms are compromised, which causes oxidative damage in the lens and eventually leads to cataract. Taken together, these findings suggest that activation and enhancement of the Nrf2-mediated antioxidant defense systems may be useful in preventing and delaying IR-induced cataract or even for other oxidative 
stress-associated diseases, in addition to the field of radiation protection.

\section{Acknowledgements}

Not applicable.

\section{Funding}

The present study was supported by financial support from the Fundamental Research Funds for the Central Universities (grant no. 3082019NT2019017). The Space Medical Experiment Project of China Manned Space Program (HYZHXM02004) and the Fundamental Research Funds for the Central Universities (Grant No. NJ2020017-3)

\section{Availability of data and materials}

The datasets used and/or analyzed during the current study are available from the corresponding author on reasonable request.

\section{Authors' contributions}

YC, JL, HZ, HL performed the experiments. JF, CX, WG designed the experiments. YC performed the data analysis and the interpretation. YC and CX confirm the authenticity of all the raw data. All authors read and approved the final manuscript.

\section{Ethics approval and consent to participate}

All procedures involving animals and the corresponding experimental protocols were approved by the Ethics Committee of Jinling Hospital (Nanjing, China) (no. 2020JLHGKJDWLS-109).

\section{Patient consent for publication}

Not applicable.

\section{Competing interests}

The authors declare that they have no competing interests.

\section{References}

1. Brennan LA, McGreal RS and Kantorow M: Oxidative stress defense and repair systems of the ocular lens. Frontiers in bioscience (Elite Ed) 4: 141-155, 2012.

2. Li Q, Bai D, Qin L, Shao M, Zhang S, Yan C, Yu G and Hao J: Protective effect of d-tetramannuronic acid tetrasodium salt on UVA-induced photo-aging in $\mathrm{HaCaT}$ cells. Biomed Pharmacother 126: 110094, 2020.

3. Spitz DR and Hauer-Jensen M: Ionizing radiation-induced responses: Where free radical chemistry meets redox biology and medicine. Antioxid Redox Signal 20: 1407-1409, 2014.

4. Liu K, Singer E, Cohn W, Micewicz ED, McBride WH Whitelegge JP and Loo JA: Time-dependent measurement of Nrf2-regulated antioxidant response to ionizing radiation Toward identifying potential protein biomarkers for acute radiation injury. Proteomics Clin Appl 13: e1900035, 2019.

5. Rafnsson V, Olafsdottir E, Hrafnkelsson J, Sasaki H, Arnarsson A and Jonasson F: Cosmic radiation increases the risk of nuclear cataract in airline pilots: A population-based case-control study. Arch Ophthalmol 123: 1102-1105, 2005.
6. Mao XW, Boerma M, Rodriguez D, Campbell-Beachler M, Jones T, Stanbouly S, Sridharan V, Wroe A and Nelson GA: Acute effect of low-dose space radiation on mouse retina and retinal endothelial cells. Radiat Res 190: 45-52, 2018.

7. Hamada $\mathrm{N}$ and Fujimichi Y: Role of carcinogenesis related mechanisms in cataractogenesis and its implications for ionizing radiation cataractogenesis. Cancer Lett 368: 262-274, 2015.

8. Markiewicz E, Barnard S, Haines J, Coster M, van Geel O, Wu W, Richards S, Ainsbury E, Rothkamm K, Bouffler S and Quinlan RA: Nonlinear ionizing radiation-induced changes in eye lens cell proliferation, cyclin D1 expression and lens shape. Open Biol 5: 150011, 2015.

9. Ainsbury EA, Barnard S, Bright S, Dalke C, Jarrin M, Kunze S, Tanner R, Dynlacht JR, Quinlan RA, Graw J, et al: Ionizing radiation induced cataracts: Recent biological and mechanistic developments and perspectives for future research. Mutat Res 770: 238-261, 2016.

10. Kensler TW, Wakabayashi N and Biswal S: Cell survival responses to environmental stresses via the Keap1-Nrf2-ARE pathway. Annu Rev Pharmacol Toxicol 47: 89-116, 2007.

11. Lee JM, Li J, Johnson DA, Stein TD, Kraft AD, Calkins MJ, Jakel RJ and Johnson JA: Nrf2, a multi-organ protector? FASEB J 19: 1061-1066, 2005.

12. Meyer LM, Lofgren S, Ho YS, Lou M, Wegener A, Holz F and Soderberg P: Absence of glutaredoxin1 increases lens susceptibility to oxidative stress induced by UVR-B. Exp Eye Res 89: 833-839, 2009.

13. Taysi S, Memisogullari R, Koc M, Yazici AT, Aslankurt M, Gumustekin K, Al B, Ozabacigil F, Yilmaz A, Tahsin Ozder H: Melatonin reduces oxidative stress in the rat lens due to radiation-induced oxidative injury. Int J Radiat Biol 84: 803-808, 2008.

14. Badhwar GD, Keith JE and Cleghorn TF: Neutron measurements onboard the space shuttle. Radiat Meas 33: 235-241, 2001.

15. Benton ER and Benton EV: Space radiation dosimetry in low-Earth orbit and beyond. Nucl Instrum Methods Phys Res B 184: 255-294, 2001.

16. Jing S, Guo H, Qi Y, Yang G and Huang Y: A portable fast neutron irradiation system for tumor therapy. Appl Radiat Isot 160: 109138, 2020.

17. Ozgen SC, Dokmeci D, Akpolat M, Karadag CH, Gunduz O, Erbas H, Benian O, Uzal C and Turan FN: The protective effect of curcumin on ionizing radiation-induced cataractogenesis in rats. Balkan Med J 29: 358-363, 2012.

18. Li C, Yang X, Xu Y, Li L and Wang Y: Cadmium detoxification induced by salt stress improves cadmium tolerance of multi-stress-tolerant Pichia kudriavzevii. Environ Pollut 242: 845-854, 2018.

19. Zhang M, Feng L, Gu J, Ma L, Qin D, Wu C and Jia X: The attenuation of Moutan Cortex on oxidative stress for renal injury in AGEs-induced mesangial cell dysfunction and streptozotocin-induced diabetic nephropathy rats. Oxid Med Cell Longev 2014: 463815, 2014.

20. Ma N, Li C, Dong X, Wang D and Xu Y: Different effects of sodium chloride preincubation on cadmium tolerance of Pichia kudriavzevii and Saccharomyces cerevisiae. J Basic Microbiol 55: 1002-1012, 2015.

21. Wang Y, Huo Y, Zhao L, Lu F, Wang O, Yang X, Ji B and Zhou F: Cyanidin-3-glucoside and its phenolic acid metabolites attenuate visible light-induced retinal degeneration in vivo via activation of Nrf2/HO-1 pathway and NF- $\mathrm{B}$ suppression. Mol Nutr Food Res 60: 1564-1577, 2016.

22. Buonanno M, de Toledo SM, Pain D and Azzam EI: Long-term consequences of radiation-induced bystander effects depend on radiation quality and dose and correlate with oxidative stress. Radiat Res 175: 405-415, 2011.

23. Tu W, Wang H, Li S, Liu Q and Sha H: The anti-inflammatory and anti-oxidant mechanisms of the Keap1/Nrf2/ARE signaling pathway in chronic diseases. Aging Dis 10: 637-651, 2019.

24. Yamaoka K, Edamatsu R and Mori A: Increased SOD activities and decreased lipid peroxide levels induced by low dose $\mathrm{X}$ irradiation in rat organs. Free Radic Biol Med 11: 299-306, 1991.

25. Macotpet A, Suksawat F, Sukon P, Pimpakdee K, Pattarapanwichien E, Tangrassameeprasert R and Boonsiri P: Oxidative stress in cancer-bearing dogs assessed by measuring serum malondialdehyde. BMC Vet Res 9: 101, 2013.

26. Sharma S, Singla N, Chadha VD and Dhawan DK: A concept of radiation hormesis: Stimulation of antioxidant machinery in rats by low dose ionizing radiation. Hell J Nucl Med 22: 43-48, 2019. 
27. Pathak CM, Avti PK, Kumar S, Khanduja KL and Sharma SC: Whole body exposure to low-dose gamma radiation promotes kidney antioxidant status in Balb/c mice. J Radiat Res 48: 113-120, 2007.

28. Ganea E and Harding JJ: Glutathione-related enzymes and the eye. Curr Eye Res 31: 1-11, 2006.

29. Donma O, Yorulmaz E, Pekel H and Suyugül N: Blood and lens lipid peroxidation and antioxidant status in normal individuals, senile and diabetic cataractous patients. Curr Eye Res 25: 9-16, 2002.

30. Ali SS, Ahsan H, Zia MK, Siddiqui T and Khan FH: Understanding oxidants and antioxidants: Classical team with new players. J Food Biochem 44: e13145, 2020.

31. Kaspar JW, Niture SK and Jaiswal AK: Nrf2:INrf2 (Keap1) signaling in oxidative stress. Free Radic Biol Med 47: 1304-1309, 2009.

32. Wang $\mathrm{W}$, Zhao $\mathrm{H}$ and Chen B: DJ-1 protects retinal pericytes against high glucose-induced oxidative stress through the Nrf2 signaling pathway. Sci Rep 10: 2477, 2020.

33. Purbey PK, Scumpia PO, Kim PJ, Tong AJ, Iwamoto KS McBride WH and Smale ST: Defined sensing mechanisms and signaling pathways contribute to the global inflammatory gene expression output elicited by ionizing radiation. Immunity 47: 421-434.e3, 2017.

34. Tsukimoto M, Tamaishi N, Homma T and Kojima S: Low-dose gamma-ray irradiation induces translocation of $\mathrm{Nrf} 2$ into nuclear in mouse macrophage RAW264.7 cells. J Radiat Res 51: 349-353, 2010.

35. McDonald JT, Kim K, Norris AJ, Vlashi E, Phillips TM, Lagadec C, Della Donna L, Ratikan J, Szelag H, Hlatky L and McBride WH: Ionizing radiation activates the Nrf2 antioxidant response. Cancer Res 70: 8886-8895, 2010.
36. Chen W, Jiang T, Wang H, Tao S, Lau A, Fang D and Zhang DD: Does Nrf2 contribute to p53-mediated control of cell survival and death? Antioxid Redox Signal 17: 1670-1675, 2012.

37. Tan XL and Spivack SD: Dietary chemoprevention strategies for induction of phase II xenobiotic-metabolizing enzymes in lung carcinogenesis: A review. Lung cancer 65: 129-137, 2009.

38. Zhao Q, Mao A, Yan J, Sun C, Di C, Zhou X, Li H, Guo R and Zhang $\mathrm{H}$ : Downregulation of Nrf2 promotes radiation-induced apoptosis through Nrf2 mediated Notch signaling in non-small cell lung cancer cells. Int J Oncol 48: 765-773, 2016.

39. Liu C, Vojnovic D, Kochevar IE and Jurkunas UV: UV-A irradiation activates Nrf2-regulated antioxidant defense and induces p53/Caspase3-dependent apoptosis in corneal endothelial cells. Invest Ophthalmol Vis Sci 57: 2319-2327, 2016.

40. Hamada N and Sato T: Cataractogenesis following high-LET radiation exposure. Mutat Res 770: 262-291, 2016.

41. Bahia S, Blais E, Murugkar S, Chauhan V and Kumarathasan P: Oxidative and nitrative stress-related changes in human lens epithelial cells following exposure to X-rays. Int J Radiat Biol 94: 366-373, 2018

42. Varma SD, Kovtun S and Hegde KR: Role of ultraviolet irradiation and oxidative stress in cataract formation-medical prevention by nutritional antioxidants and metabolic agonists. Eye Contact Lens 37: 233-245, 2011.

43. Tülüce Y, Ozkol H and Koyuncu I: Photoprotective effect of flax seed oil (Linum usitatissimum L.) against ultraviolet C-induced apoptosis and ox idative stress in rats. Toxicol Ind Health 28: 99-107, 2012.

This work is licensed under a Creative Commons Attribution-NonCommercial-NoDerivatives 4.0 International (CC BY-NC-ND 4.0) License. 